\title{
Display of spatial coherence of light in interference experiments: laboratory works and demonstrations
}

\section{Vladimir Ryabukho, Ol'ga Perepelitsina, Valery Tuchin}

Vladimir P. Ryabukho, Ol'ga A. Perepelitsina, Valery V. Tuchin, "Display of spatial coherence of light in interference experiments: laboratory works and demonstrations," Proc. SPIE 4588, Seventh International Conference on Education and Training in Optics and Photonics, (28 May 2002); doi: 10.1117/12.468741

SPIE Event: Education and Training in Optics and Photonics 2001, 2001, Singapore, Singapore 


\title{
Display of spatial coherence of light in interference experiments: laboratory works and demonstrations
}

\author{
Vladimir P.Ryabukho, Ol'ga A.Perepelitsina and Valery V.Tuchin \\ Saratov State University, Saratov, RU 410026
}

\begin{abstract}
In the paper two different approaches for spatial coherence measurement are discussed. The usage of a special optical element (specklegram of shift) in the scheme of Young interferometer essentially raises a relative aperture of the optical device and allows one by an evident way to study the spatial coherence of light and to measure radius of spatial coherence. It is shown with use of the Michelson interferometer that the consideration of mutual spatial shift of interfering fields allows one to connect directly the spatial distribution of fringe visibility in the area of their localization with the function of a spatial coherence. The theoretical estimations and experimental results for longitudinal distribution of fringe visibility in the area of localization in the Michelson interferometer with an extended source of white light are given. The description of laboratory work and the demonstration instruments are discussed.
\end{abstract}

Keywords: interference, coherence, Young and Michelson interferometers, specklegram, fringe visibility, localization.

\section{INTRODUCTION}

The spatial coherence of light is one of the important and complicated notions of the wave optics. Usually the spatial coherence of light is discussed on an example of interferometers with the division of a wave front, mostly the Young interferometer ${ }^{1-10}$. However, the classical two-slit statement of the Young interferometer has extremely small apertures and this scheme is practically none applicable neither to lecture demonstrations, nor for performance of laboratory work on study of influence of spatial coherence on contrast of formed interference fringes. Because of major light losses the Young interferometer can practically be realized in the frame of laboratory work only with use of enough power laser radiation. The simple experimental research of influence of spatial coherence of light on interference fringes contrast is possible with use of a special optical element in Young interferometer, for example, specklegram of shift ${ }^{11}$. This optical element essentially raises an aperture of the optical device and allows one to study the spatial coherence of light and to measure the radius of spatial coherence by an evident way.

The clear display of spatial coherence of light is also possible in interferometers with division of amplitude. The consideration of mutual spatial shift of interfering light fields allows for directly connect spatial distribution of fringe visibility in the area of their localization with the function of a spatial coherence. The general analysis of the mutual shift of fields can be rather complicated, however in the schemes with an optical wedge this analysis is enough simple and appropriate for demonstration of display of a spatial coherence in interference experiment. For example, with use of the Michelson interferometer it is not difficult to show how the spatial coherence of light is manifested in the fringes localization.

\section{YOUNG INTERFEROMETER}

The classical scheme of Young interferometer is presented in Fig.1,a. In Ref.1 more convenient Young scheme with perfect overlapping of diffracted light beams (Fig. 1,b) is given. Two important features of this scheme should be noted. The first one is that for enough thin lens and enough large distance from the screen to the observation plane $z$ the period of fringes $\Lambda$ is defined by the expression $\Lambda=\bar{\lambda} z / \Delta x\left(1+\Delta z / z_{s}\right)$, where $\Delta x$ is the distance between centers of holes, $\bar{\lambda}$ is the average wavelength, $z_{s}$ is the distance from the source to the screen, $\Delta z$ is the distance from the lens to the screen. The second feature is that the Young fringes are localized in the plane, which is optically conjugated with source $S$. Therefore, the light field in observation plane is defined by Fourier transform of field that is formed immediately behind the screen with the holes ${ }^{12,13}$. The position of interference fringes does not depend on the position 
of holes in the screen with respect to optical axis because of the lens tautochronism. Therefore, the bright interference fringe of zero order is formed in the center of the source image.
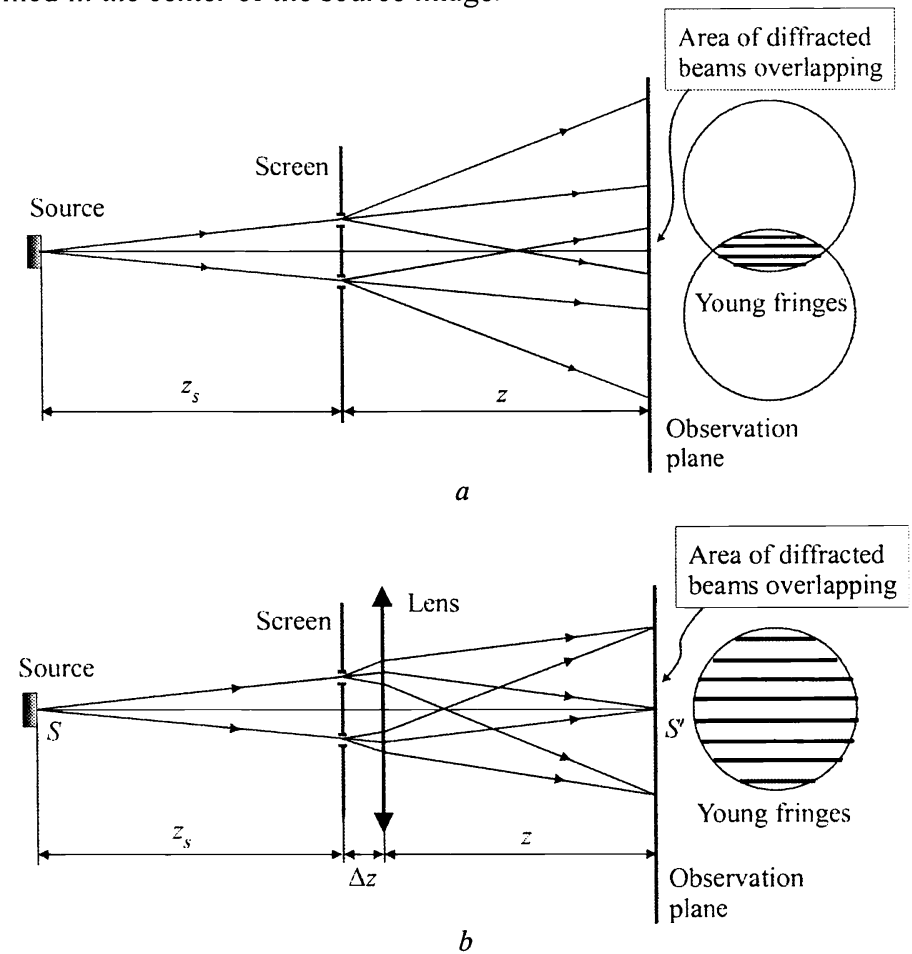

Fig. 1. (a) Classical scheme of Young interferometer; $(b)$ Young interferometer with using of collecting lens

If there is the set of pairs of holes with identical orientation and form and with equal distance $\Delta x$ in the screen, the set of the corresponding interference Young fringes will be superposed against each other without mutual shift, increasing the light intensity in bright fringes.

It is possible to produce such screen with a set of pairs of holes with the help of a photolithography. However such method is enough laborious. The technologically simple method of obtaining the required screen can be realized by two-exposition photographic recording of speckle-pattern formed in a scattered laser light ${ }^{14-16}$. The optical scheme, which can be used for these purposes, is given in Fig.2. The rather wide laser beam incidents on a ground glass, which image is twice photographed on a holographic plate. This plate is fixed on the table with the possibility of its transverse micrometrical displacement. Fig. 3 demonstrates the interference Young fringes formed in the diffraction halo of the farzone field when narrow laser beam incidents on the specklegram of shift.

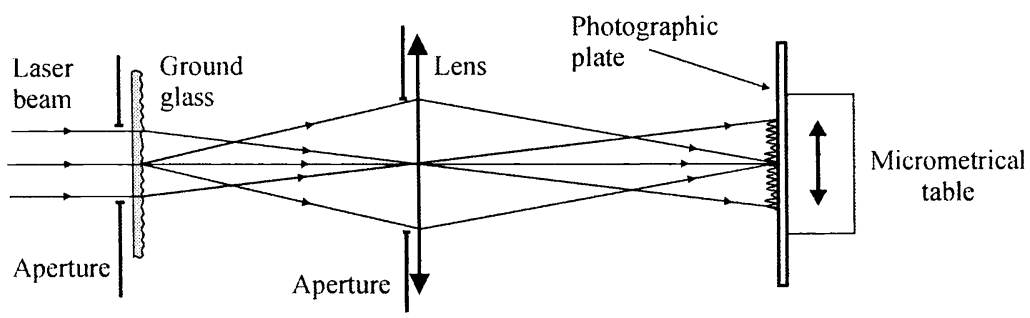

Fig.2. The scheme of recording of two-exposition specklegram of shift

If the round aperture of imaging lens is used on the stage of recording of two-exposition specklegram of shift (Fig.2), there is a great difference of light intensity at center and on edges of diffraction halo (Fig. $3, a, b$ ). For diminution of this 
difference and for more noticeable outline of diffraction halo it is possible to use the ring aperture of imaging lens for recording of specklegram ${ }^{16}$. In Fig.3,c,d there are the patterns of interference Young fringes obtained by use of the specklegrams recorded by such technique.

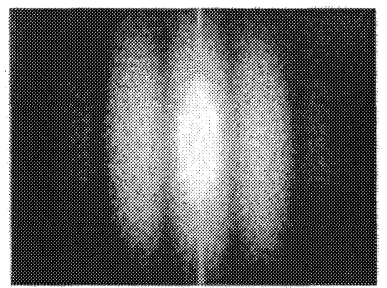

$a$

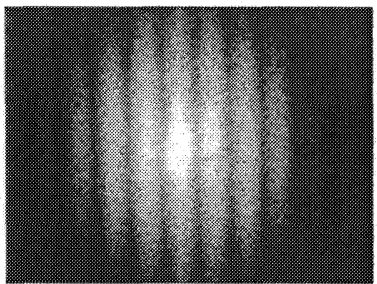

$b$

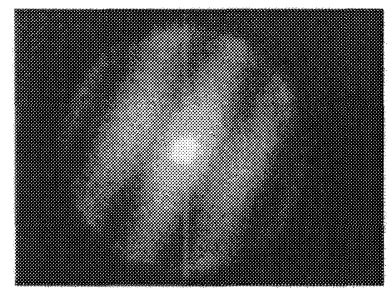

$c$

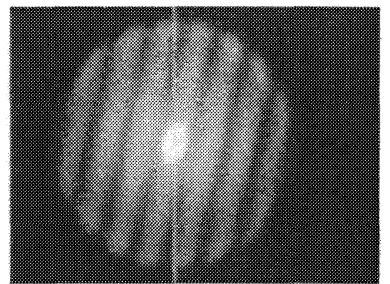

$d$

Fig.3. The interference Young fringes in far-zone of laser beam diffraction on different specklegrams of transverse shift obtained by use of round $(a, b)$ and ring $(c, d)$ apertures of imaging lens on the stage of specklegram recording (see Fig.2)

The clearly visible interference Young fringes are observed in diffraction halo at distance of $\sim 1 \mathrm{~m}$ from the specklegram of shift when it is illuminated by the narrow laser beam. At this distance the diameter of diffraction halo is $B \approx 2 \lambda z / \varepsilon_{\perp} \approx 40 \mathrm{~cm}$, when the mean speckle size on the specklegram is $\varepsilon_{\perp} \approx 3 \mu \mathrm{m}$. When the laser power is equal to $P \approx 10 \mathrm{~mW}$ the fringe pattern is seen well in the large lecture-hall.

To demonstrate the influence of spatial coherence of light on Young fringes contrast it is better to use the scheme, presented in Fig. 1,b. The change of fringe contrast is observed in the lecture-room with the sufficient darkening. If the eye lens is used as a focusing lens, the visual power efficiency of the scheme increases essentially, since the interference Young fringes forms directly on the retina of the observer (Fig.4). Therefore, the observation of the influence of the spatial coherence on the interference fringe contrast is possible in the lecture-room without darkening. For this it is enough to have two light sources with differed noticeably sizes of luminous elements in the lecture-room (for example, the incandescent lamp with visible filament and the incandescent lamp with opal bulb). In Fig.5 the photograph of interference fringes of different contrast is presented. These fringes are seen in the halo of illuminating lamps placed on the ceiling of the Big Physical Lecture-Hall of Saratov State University, when lamps are observed through the specklegram of shift.

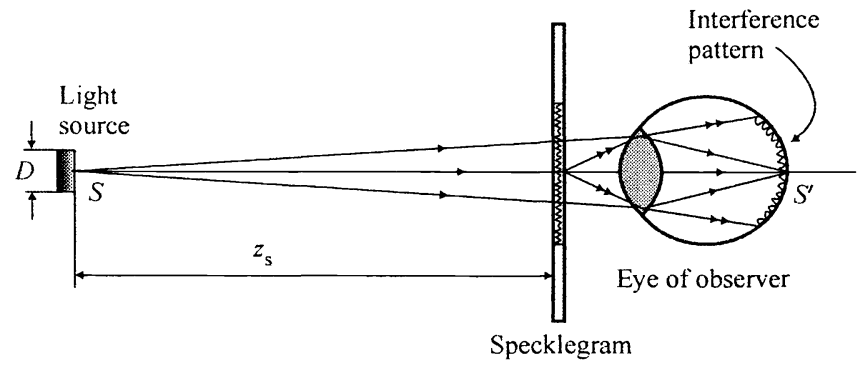

Fig. 4. The optical scheme for directly forming of interference Young fringes in the retina of observer's eye

On the basis of the optical scheme presented in Fig. 1 and Fig.4, it is possible to make of laboratory work for study of influence of the degree of spatial coherence of extended light source on the visibility of the interference fringes ${ }^{17}$.

Two modifications of this work are possible. If there are available several specklegrams with different transverse shift $\Delta x$ of speckle patterns, the specklegram for which the Young fringes disappears for given angular size of light source should be selected. In this case the transverse shift $\Delta x$ is approximately equal to the spatial coherence radius $\rho_{c}$ in the specklegram plane $\Delta x \approx \rho_{c}$. For this specklegram the angular period of Young fringes $\Lambda_{\alpha}$ is defined by the source 
with less angular sizes and $\rho_{c} \approx \Delta x=\bar{\lambda} / \Lambda_{\alpha}$. From calculated value $\rho_{c}$ the angular size of light source can be determined using the expression $\theta \approx \bar{\lambda} / \rho_{c}$ and compared with the directly measured value of $\theta=D / z_{s}$.

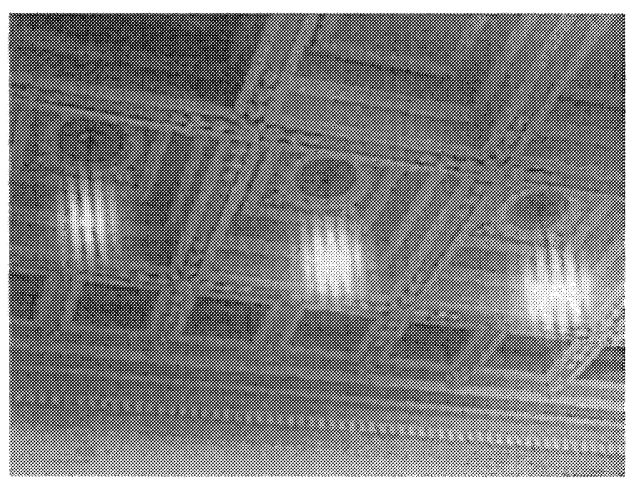

Fig.5: The interference Young fringes of different contrast seen when lamps are observed through the specklegram of shift (the lamp with visible filament is found on the left, two lamps with scattered bulbs is found on the right)

In the second modification it is necessary to use one specklegram and the light source, which transverse sizes can be continuously changed during experiment, for example, the illuminator with ground glass and iris diaphragm. The variation of Young fringes contrast is observed by changing the source light diameter (Fig.6). The fringes disappear when $\Delta x \approx \rho_{c}$. In this experiment the quantity $\Delta x$ is determined by the measurement of the fringe spacing $\Lambda$, when the source size is relatively small and the fringes are clearly seen. For this measurement it is necessary to install the scale in the light source plane. Then in the visible field of observer the interference fringes and the scale for determination of fringes period $\Lambda$ should be seen simultaneously. By knowing the distance $z_{s}$ from the source to the specklegram the fringe spacing $\Lambda$, the quantity of shift $\Delta x=\bar{\lambda} z_{s} / \Lambda$ and, consequently, the radius of light coherence $\rho_{c} \approx \Delta x$ can be determined. The determined value of $\rho_{c}$ can be compared with the calculated value, $\rho_{c} \approx \bar{\lambda} z_{s} / D$, where $D$ is the source diameter.

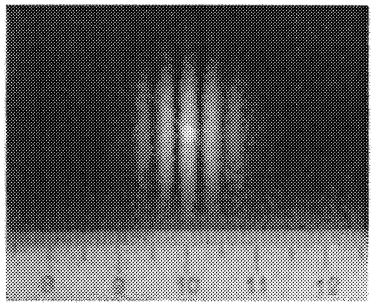

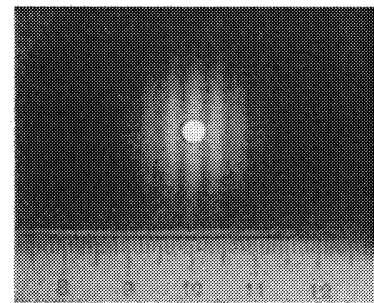

$b$

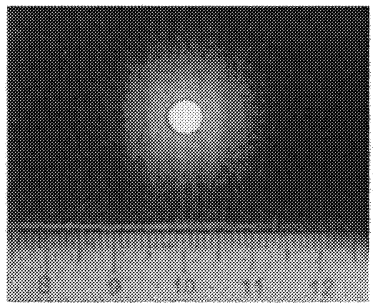

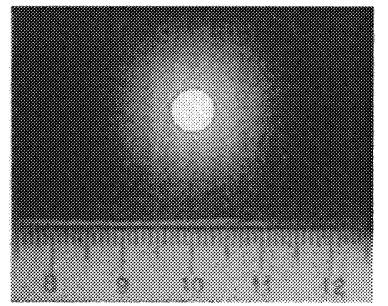

$d$

Fig.6. The decrease of contrast of interference Young fringes observed in laboratory experiment with increasing the light source size: $a$ and $b-\rho_{c}>\Delta x ; c-\rho_{c} \approx \Delta x ; d-\rho_{c}<\Delta x$

In conformity with the Van Cittert-Zernike theorem for the uniform luminous of the round light source the normalized spatial transverse coherence (the degree of coherence) of light is determined by the expression ${ }^{1-4}$ :

$$
\gamma_{12}(\Delta x)=\frac{2 J_{1}(u)}{u}, u=\frac{\pi D \Delta x}{\bar{\lambda} z_{s}},
$$

where $J_{1}(u)$ is the Bessel function of the first order. The curve of the function $\gamma_{12}(u)$ is shown in Fig.7. 


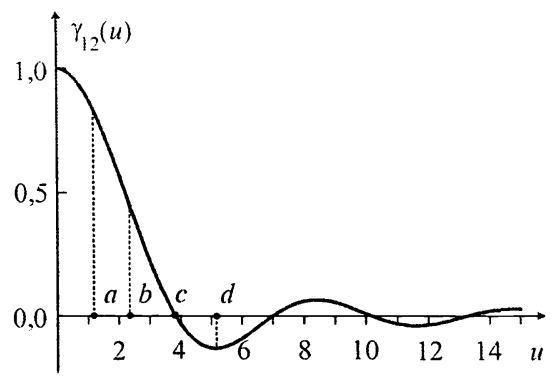

Fig.7. The function of spatial transverse coherence of light of the circle-shaped thermal source

The photographs of interference fringes demonstrated in Fig. 6 are obtained for the points $a, b, c, d$ shown in Fig.7. For point $d(u \approx 5,15)$ the coherence degree has the negative value $\gamma_{12} \approx-0,132$. From the physical viewpoint this is equivalent to the additional phase difference $\pi \mathrm{rad}$ between the interfering waves. Therefore, in Young pattern the reversal contrast fringes are observed, the central interference fringe of the zero-order becomes dark (Fig.6,d) ${ }^{1,4}$.

\section{MICHELSON INTERFEROMETER}

It is known that the interference fringes formed in a partially coherence light are spatially localized ${ }^{1,5}$. Due to this effect the spatial or temporal coherence of light is clearly manifested.

In Fig.8, $a$ the scheme of Michelson interferometer with a flat mirrors and an extended polychromatic source of light is shown. For observing the interference of light waves reflected by mirrors $M_{1}$ and $M_{2}$ it is necessary to provide the optical path difference of these waves in the interferometer arms $\Delta$ smaller than the coherence length of light $\Delta<l_{c}$. Suppose the interferometer arms are equalized and $\Delta=0$ for central light ray. For observation of interference fringes with a finite width it is necessary to create the changing space path difference $\Delta \Phi(\vec{r})$ between interfering waves. In Michelson interferometer this is possible by making the tilt of a mirror at a small angle. As a consequence the air wedge between the mirrors images is formed and parallel strait fringes are observed '.
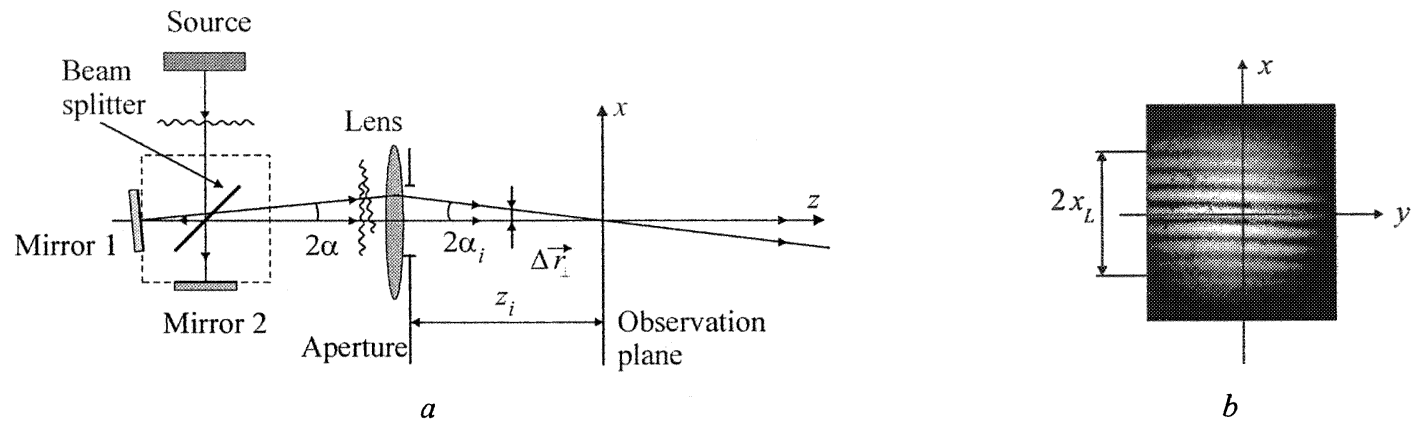

Fig.8: (a) The Michelson interferometer for observation of effects of temporal and spatial light coherence in the interference fringes visibility. $(b)$ The interference fringes observed in white light in the plane of real image of the mirrors

Besides the path difference, the tilt of the mirror results in an inclination of the reflected wave at the angle $2 \alpha$ around the tilt axis. Therefor the reflected wave suffers a transverse shift $\Delta r_{\perp} \approx 2 \alpha z$, where $z$ is a distance from the mirror. Thus the two identical and completely mutually coherent waves are propagating with the path difference $\Delta \Phi(\vec{r})$ and with mutual transverse shift $\Delta r_{\perp}=2 \alpha z$

We can write with regard to phase difference and the mutual shift of the light fields in interferometer output in scalar approximation:

$$
E_{2}(\vec{r}, t)=K \cdot E_{1}(\vec{r}-\Delta \vec{r}, t-\Delta t) \exp [i \Delta \Phi(\vec{r})]
$$


where $\Delta t$ is the mutual time delay of waves in interferometer arms, $\Delta \vec{r}$ is the mutual shift of fields, $K$ is the coefficient of amplitude division of initial wave in interferometer. The Eq.(2.1) means that the interference fields are identical with respect to their spatial and temporal amplitude-phase structure. Therefore they are completely coherent, if the disturbances causing by the fields are considered at the corresponding points. Otherwise we consider the light oscillations at point $\vec{r}$ for field $E_{1}(\vec{r}, t)$ and at point $(\vec{r}+\Delta \vec{r})$ for the field $E_{2}(\vec{r}, t)$, correspondingly.

The mutual shift of these identical fields results in a certain degree of mismatching for overlapping light oscillations because of their limited degree of spatial coherence. This causes the decrease of contrast of observed interference fringes.

The shift between the fields introduced by interferometer is changed in space, $\Delta \vec{r}=\Delta \vec{r}(\vec{r})$. From the viewpoint of the influence of this shift on fringe contrast the relation between the shift $|\Delta \vec{r}|$ and $\rho_{c}$ in the direction of the shift is important. If $|\Delta \vec{r}|<<\rho_{c}$ then it is possible to consider $\Delta r \approx 0$. Consequently, the disturbances caused by fields are mutually coherent and the fringes of maximum contrast are observed. If the value $\Delta \vec{r}$ will exceed the spatial coherence radius $|\Delta \vec{r}|>\rho_{c}$, the mutual coherence of fields is loosing and the fringes disappear. If the mutual time delay of waves in interferometer is $\Delta t=0$ (the arms are equaled), the fringe contrast is determined by the normalized function of spatial coherence $\gamma_{12}(\Delta \vec{r})$ for the present direction and for the mutual fields shift $\Delta \vec{r}$

$$
\gamma_{12}(\Delta \vec{r}, \Delta t)=\frac{\left\langle E_{1}(\vec{r}, t) E_{2}^{*}(\vec{r}, t)\right\rangle}{\left[I_{1}(\vec{r}) I_{2}(\vec{r})\right]^{1 / 2}}=\frac{\left\langle E_{1}(\vec{r}, t) E_{1}^{*}(\vec{r}-\Delta \vec{r}, t-\Delta t\rangle\right.}{\left[I_{1}(\vec{r}) I_{1}(\vec{r}-\Delta \vec{r})\right]^{1 / 2}} .
$$

Let as determine the location and the extension of the area of fringes localization and spatial distribution of the fringe visibility. The interference fringes of maximum contrast are observed in real image plane of interferometer mirrors, where the mutual shift of fields is practically absent, $\Delta r_{\perp}=0$ (Fig. 8,a). Because of the mutual inclination of mirrors the path difference $\Delta$ of waves in the image plane is changed in the direction $x$, which is perpendicular to the fringes. Therefore the area of fringes localization in this direction can be limited if the path difference $\Delta$ will reach the coherence length $l_{c}, \Delta \geq l_{c}$ (Fig.8,b). If the fringes spacing in the image plane $\Lambda_{i}=\bar{\lambda} / 2 \alpha_{i}$, where $2 \alpha_{i}$ is the angle of mutual inclination of the wave front of the fields in the image plane, it is possible to write the expression for the extent of the area of fringes localization in this plane:

$$
\Delta=l_{c} \leftrightarrow \frac{\bar{\lambda}}{\Lambda_{i}} x_{L}=\frac{\bar{\lambda}^{2}}{\Delta \lambda} \leftrightarrow 2 x_{L}=2 \frac{\bar{\lambda}}{\Delta \lambda} \Lambda_{i}
$$

The extent of the area of fringes localization Along optical axis of the system is limited by the spatial coherence of light, since the interference fields outside of mirrors image plane acquire the mutual transverse shift, which is linearly increased at distance from the image equal to $\Delta r_{\perp}=2 \alpha_{i} l=\bar{\lambda} l / \Lambda_{i}$, where $l$ is the distance from image to considered field section. When the fields shift at the present field section is equal to the spatial coherence radius $\rho_{c}$ or is larger then $\rho_{c}$, the interference fringes disappear. Thus the interference fringes in the image space acquire the clear localization with maximum contrast in the plane of mirrors image.

It is possible to consider the exit pupil of optical system for the image space as the secondary extended incoherent source of light. If the angular sizes of the real source are greater than the angular aperture of imaging system $\theta>\theta_{a}$, it is necessary to use the full aperture of exit pupil as the secondary source. Let the optical system has the rectangular aperture, which is located along interference fringes and has the width $d$. We can obtain the relation for the degree of light spatial coherence in the image space and, consequently, the fringe contrast with use of the Van Cittert-Zernike theorem:

$$
V(z)=\gamma_{12}\left(\Delta r_{\perp}\right)=\operatorname{sinc}\left(\frac{\pi d}{\bar{\lambda} z}\left|\Delta \vec{r}_{\perp}\right|\right)=\operatorname{sinc}\left(\frac{\pi d}{\bar{\lambda} z}\left(z-z_{i}\right) \frac{\bar{\lambda}}{\Lambda_{i}}\right)
$$


where $z$ is the distance from exit pupil of the system to the considered section of field, $z_{i}$ is the distance to mirrors image plane.

The boundaries of localization area are determined by the condition of equality to zero of sinc-function: $\pi d\left(1-z_{i} / z\right) / \Lambda_{i}= \pm \pi$. Hence, the two coordinates for the boundaries of localization area are:

$$
z_{1}=z_{i} /\left(1+\frac{\Lambda_{i}}{d}\right), \quad z_{2}=z_{i} /\left(1-\frac{\Delta_{i}}{d}\right)
$$

The absolute values of distances from the image plane to the boundaries of localization area are $l_{1}$ and $l_{2}$ and the general extension of localization area is determined by the relation:

$$
L=l_{1}+l_{2}=z_{i} \frac{\Lambda_{i} / d}{1+\Lambda_{i} / d}+z_{i} \frac{\Lambda_{i} / d}{1-\Lambda_{i} / d}=2 z_{i} \frac{\Lambda_{i} / d}{1-\left(\Lambda_{i} / d\right)^{2}} .
$$

The asymmetry of fringe localization area has enough simple explanation. The spatial coherence radius of light in the image area, $\rho_{\perp}$, is determined by Eq.4, when the first sinc-function is equal to zero: $\pi d \Delta r_{\perp} / \bar{\lambda} z=\pi \rightarrow \rho_{\perp}=\bar{\lambda} z / d$. Thus at distance range from image plane to exit pupil the mutual shift of interference fields $\Delta r_{\perp}$ increases whereas the radius of spatial coherence decreases. At distance range from image plane to far zone both coherence radius and shift of fields increase. Hence, in this direction the mutual coherence of the fields is kept for greater distance, than in the direction to the lens pupil.
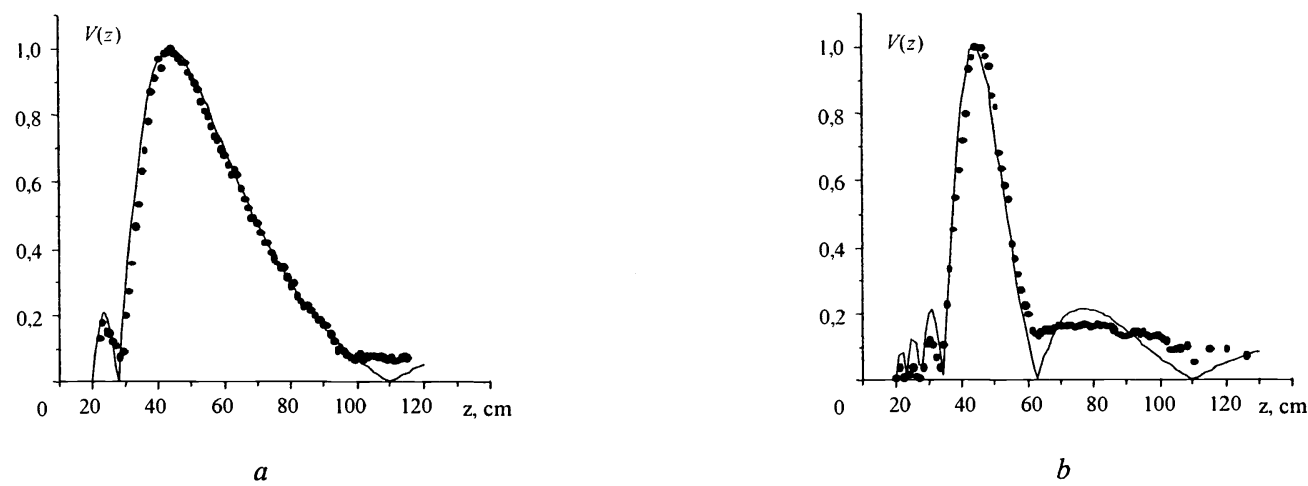

Fig.9: The mutual spatial coherence degree of interfering waves and, correspondingly, the fringe contrast in the area of their localization with different ratio of pupil diameter of the optical system and fringes spacing $d / \Lambda_{i}$ as a function of distance from the lens pupil (the experimental data and the theoretical curves): $\mathrm{a}-d / \Lambda_{i}=1.67 ; \mathrm{b}-d / \Lambda_{i}=3.33$

Fig. 9 shows the experimental data and the theoretical curves for variation of fringe contrast along optical axis of the system obtained with different ratio $d / \Lambda_{i}$ (with different fringe spacing $\Lambda_{i}$ and constant width of exit pupil). At $d / \Lambda_{i} \rightarrow 1$ the distance $l_{2} \rightarrow \infty$ and the localization area is limited practically only in a direction to the lens.

In Eq.(1.4) the fringe contrast is not equal to modulus of the coherence degree $\gamma_{12}\left(\Delta \vec{r}_{\perp}\right)$. The sign variation of the coherence degree $\gamma_{12}$ is necessary to interpret as an effect of fringe contrast reversal. This effect is well observed in the experiment.

\section{CONCLUSION}

The usage of the special screen with a set of pairs of identical holes or of transparent spots (specifically, the usage of a specklegram of shift) essentially simplifies an arrangement of interference experiment of Young. It is got possible the 
display of Young fringes at laser radiation in not darkening lecture-room and the organization of laboratory work to study the effect of spatial light coherence of broad thermal sources using classical Young interferometer. The more complete experiment on study of spatial light coherence in Young scheme intend to measure a fringe contrast $V$ depending on distance between the holes $\Delta x$ and to determine a function of spatial light coherence $\left|\gamma_{12}(\Delta x)\right|=V(\Delta x)$.

Such experiment is more difficult. However, the usage of video camera, personal computer and corresponding software for image processing will allow one to make quickly measurements of fringe contrast.

On the basis of Michelson interferometer the laboratory work to study the effects of manifestation of spatial and temporal coherence of light, to determine the width of spectral profile of radiation, to study of depth of localization area of fringes as function of spatial light coherence parameters is made possible ${ }^{18}$. The adjustment and service of Michelson interferometer in accordance with scheme shown in Fig. 8 is not difficult. Suggested interferometer allows one to obtain the interference pattern in a white light without any special vibration protection and demonstrate the interference pattern in the large lecture-hall. The Michelson interferometer has a comparatively small dimensions $300 \times 200 \times 100 \mathrm{~mm}$, a good light-gathering power, sufficed for formation of interference pattern with diameter $\sim 1 \mathrm{~m}$ in white light of a halogen lamp.

The laboratory work on the basis of Michelson and Young interferometers shall simplify the study of a rather difficult for learning part of physical optics "The spatial coherence of light".

\section{ACKNOWLEDGEMENTS}

The research described in this publication was made possible in part by the Award No REC-006 of the U.S. Civilian Research \& Development Foundation for the Independent States of the Former Soviet Union (CRDF) and by grant No. 00-15-96667 of the "Leading Scientific Schools" Program of the Russian Basic Research Foundation.

\section{REFERENCES}

1. M.Born and E.Volf, Principles of optics. Pergamon Press, N.Y., 1964.

2. M.Françon, S.Slansky, Cohérence in optique. Éditions du centre national de la recherche scientifique, Paris, 1965.

3. S.A.Akhmanov, Yu.E.Dyakov, A.A.Chirkin, Introduction to statistical radio-physics and optics. Nauka, Moscow, 1981 (in Russian).

4. J.W.Goodman, Statistical optics. John Wiley \& Sons, N.Y., 1985.

5. G.S.Landsberg, Optics. Nauka, Moscow, 1976 (in Russian).

6. I.V.Savel'ev, Course of general physics. Vol.2. Electricity and Magnetism. Waves Optics. Nauka, Moscow, 1978 (in Russian).

7. N.I.Kaliteevsky, Wave optics. Visshaya Shkola, Moscow, 1995 (in Russian).

8. A.N.Matveev, Optics. Visshaya Shkola, Moscow, 1985 (in Russian).

9. E.I.Butikov, Optics. Visshaya Shkola, Moscow, 1986 (in Russian).

10. S.A.Akhmanov, S.Yu.Nikitin, Physical optics. Published by Moscow University, Moscow, 1998 (in Russian).

11. N.Takai, H.Amber, T.Asakura, "Spatial coherence measurements of quasi-monochromatic thermal light using double-exposure specklegrams", Opt. Commun. 60, pp.123-127, 1986.

12. J.W.Goodman, Introduction to fourier optics. McGraw - Hill Book Company, N.Y., 1968.

13. R.J.Collier, C.B.Burckhardt, L.H.Lin, Optical holography. Academic Press, N.Y., 1972.

14. M.Françon, La granularité laser (spekle) et ses applications en optique. Masson, Paris, 1978.

15. R.Jones, C.Wykes, Holographic and speckle interferometry Cambridge University Press, Cambridge, 1983.

16. I.S.Klimenko, Holography of focused images and speckle-interferometry. Nauka, Moscow, 1988 (in Russian).

17. V.P.Ryabukho, O.A.Perepelitsina, "Study of manifestation of spatial light coherence in Young's scheme in demonstration and laboratory experiments", Physics in Higher Education. 7, N 4, 2001 (in Russian).

18. V.P.Ryabukho, O.A.Perepelitsina, "On localization of interference fringes in partial coherence light", Physics in Higher Education. 7, N 2, pp.15-27, 2001 (in Russian). 